\title{
NOTE FAUNISTIQUE SUR LES PLÊCOPTẼRES DU MASSIF CENTRAL
}

\author{
par C. Berthélemy.
}

Nous apportons ici des compléments à la liste des Plécoptères du Massif Central récemment publiée par J. Aubert [1963 a]. Il s'agit, pour une bonne part, d'exemplaires provenant de régions différentes de celles qu'a visitées M. Aubert, en particulier de la bordure sud-ouest du Massif et de la Montagne Noire. Nous y avons ajouté quelques localités du Bas-Languedoc qui étaient restées inédites ou étaient dispersées dans plusieurs publications. Les dates de captures antérieures à 1950 correspondent à des animaux examinés dans la Collection DEsPax. Les captures postérieures ont été faites par $M^{\text {He }}$ Spychalowicz et MM. H. Décamps et Y. Coineau. Nous leur adressons nos plus vifs remerciements, ainsi qu'à

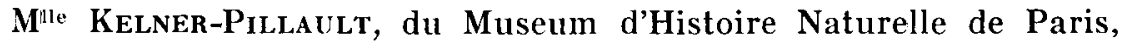
grâce à qui nous avons pu étudier la Collection DEsPax dans d'excellentes conditions, et à MM. D. E. Kimmins et P. Brinck qui ont bien voulu nous envoyer des Nemoura de Grande-Bretagne et de Suède.

\section{LISTE DES LOCALITES}

Pour faciliter les comparaisons, nous avons employé le même procédé qu'AuBErT [1963 a] : les noms en italiques sont ceux qui sont utilisés dans la liste des captures pour désigner les stations. Les lettres $\mathbf{N}$. et $\mathbf{D}$. suivies d'un numéro désignent les routes nationales et départementales; les localités et lieux-dits figurent sur les cartes routières Michelin; les altitudes non précédées de « vers » ont été obtenues à partir des cartes au $1 / 50000^{\mathrm{e}}$ publiées par l'I.G.N.

Haute-Vienne : Pierre-Buffière, vers $300 \mathrm{~m}$; Saint-Vitte-surBriance, vers $300 \mathrm{~m}$.

Puy-de-Dôme : Clermont-Ferrand, vers $500 \mathrm{~m}$; Royat, vers $500 \mathrm{~m}$; Ceyrat, vers $600 \mathrm{~m}$; la Couze Pavin (riv.), près de Besseen-Chandesse, vers $1000 \mathrm{~m}$; l'Allier à Vic-le-Comte, vers $300 \mathrm{~m}$; ruisseau du Lot, près de Saint-Saturnin, vers $700 \mathrm{~m}$; le Mont Dore, vers $1500 \mathrm{~m}$. 
Cantal : Le Lioran, vers $1200 \mathrm{~m}$; Murat, vers $800 \mathrm{~m}$; ruisseau de Prat-de-Bouc, vers $1000 \mathrm{~m}$; cirque du Brezons, vers $1500 \mathrm{~m}$; vallée de la Rhue de Cheylade, vers $1000 \mathrm{~m}$; la Jordanne (riv.), près de Saint-Julien-de-Jordanne, vers $1000 \mathrm{~m}$; la Jordanne, près de Velzic, vers $800 \mathrm{~m}$; la Jordanne, près de Saint-Simon, vers $700 \mathrm{~m}$; la Jordanne, près d'Aurillac, vers $700 \mathrm{~m}$; la Cère (riv.) à Carbonnat; la Cère, à Arpajon-sur-Cère, vers $700 \mathrm{~m}$.

Lozère : Monts d'Aubrac, cascade de Dérols, vers $1000 \mathrm{~m}$; ruisseau près du lac de Salhiens, vers $1000 \mathrm{~m}$; ruisseau près du lac de Saint-Andéol, vers $1000 \mathrm{~m}$; Mende, vers $300 \mathrm{~m}$.

Aveyron : Rodez, vers $600 \mathrm{~m}$; Millau, vers $500 \mathrm{~m}$.

Tarn-et-Garonne : Miramont-de-Quercy, vers $200 \mathrm{~m}$.

Tarn : le Céret 1 (riv.), N. 88, vers $500 \mathrm{~m}$; le Céret 2, près de Monestiès, vers $200 \mathrm{~m}$; Senouillac, vers $150 \mathrm{~m}$; Gaillac, vers $100 \mathrm{~m}$; Albi, vers $200 \mathrm{~m}$; l'Agout (riv.) près de Castres, vers $150 \mathrm{~m}$; Anglèsdu-Tarn, vers $700 \mathrm{~m}$; le Sor (riv.), près d'Arfons, vers $600 \mathrm{~m}$; le Sor, D. 44, $300 \mathrm{~m}$; affluent du Sor, un peu en amont de Sorèze, D. $45,500 \mathrm{~m}$; le Taurou (riv.), S.-W. de Dourgne, D. 85, $200 \mathrm{~m}$; ruisseau au S. de Massaguel, D. 14, $440 \mathrm{~m}$; le Pas-du-Sant, à $2 \mathrm{~km}$ au S. d'Escoussens, D. 16, $440 \mathrm{~m}$; Forêt de Montaud, vers $600 \mathrm{~m}$; ruisseau de Montimont $1,9 \mathrm{~km}$ au $\mathrm{S}$. de Labruguière, D. $56,500 \mathrm{~m}$; ruisseau de Montimont $2,5 \mathrm{~km}$ au $\mathrm{S}$. de Labruguière, D. $56,320 \mathrm{~m}$; affluent de l'Arnette, au N. des Rousses, $660 \mathrm{~m}$; l'Arnette 2 (riv.), au N.-W. de Roquerlan, D. 54, $600 \mathrm{~m}$; l'Arnette 3, $480 \mathrm{~m}$; l'Arnette, au S. de Mazamet, D. $54,480 \mathrm{~m}$; affluent de l'Arnette, au N. de Roquerlan, $660 \mathrm{~m}$; ruisseau $1 \mathrm{~km}$ au ${ }_{n} \mathrm{~S} . \mathrm{W}$. de la Maison du Triby, $800 \mathrm{~m}$; ruisseau d'Albine 1, au $\mathrm{N}$. de Lébrat, N. 620, $800 \mathrm{~m}$; ruisserau d'Albine $2,2 \mathrm{~km}$ en aval du précédent, N. 620, D. $88,780 \mathrm{~m}$; ruisseau d'Albine 3, D. $88,680 \mathrm{~m}$; ruisseau d'Albine 4, D. 88, $640 \mathrm{~m}$; Forêt de Nore, au N. de SaintAmans-Soult, vers $400 \mathrm{~m}$.

Haute-Garonne : Saint-Ferréol, vers $300 \mathrm{~m}$.

Aude : affluent du Fresquel, $2 \mathrm{~km}$ à l'E. de Saint-Papoul, D. 126, $150 \mathrm{~m}$; le Tenten (riv.), au N. du château de Ferrals, D. 103, $229 \mathrm{~m}$; affluent de la Montagne, en forêt de Ramondens, $840 \mathrm{~m}$; le Lampy, vers $600 \mathrm{~m}$; la Rigole (riv.) à l'W. de la Galaube, D. 53, $660 \mathrm{~m}$; forêt de la Loubatière, vers $700 \mathrm{~m}$; l'Alzeau (riv.), à l'entrée de Montolieu, D. $8,200 \mathrm{~m}$; la Rougeanne (riv.), au S. de Montolieu, $150 \mathrm{~m}$; la Dure (riv.), au N.-W. de Brousse, D. 109, $395 \mathrm{~m}$; la Dure, à l'E. de Cuxac-Cabardès, D. 62, $518 \mathrm{~m}$; l'Orbeil (riv.) à la sortie de Mas-Cabardès, D. 101, $295 \mathrm{~m}$; l'Orbeil, au S.-E. du bois des Gramontes, D. 101, N. 118, $750 \mathrm{~m}$; l'Arnette 1 (riv.) près de la source, D. 87, $1000 \mathrm{~m}$; ruisseau de Ganganel, à l'W. de Cabrespine, D. $112,329 \mathrm{~m}$; le Clamoux 1 (riv.), D. $112,300 \mathrm{~m}$; le Cla$\operatorname{moux} 2$, N. 620, $170 \mathrm{~m}$; le Cros (riv.), $2 \mathrm{~km}$ au N.-W. de Trausse, 
D. $115,160 \mathrm{~m}$; l'Argent-Double (riv.), $1,5 \mathrm{~km}$ au N. de CaunesMinervois, N. 620, $238 \mathrm{~m}$; l'Argent-Double, $3 \mathrm{~km}$ au S. de Citou, N. 620, $294 \mathrm{~m}$; l'Argent-Double, $1 \mathrm{~km}$ au $\mathrm{S}$. de Lespinassière, N. 620, $400 \mathrm{~m}$; affluent de l'A rgent-Double, à l'W. de Lespinassière, D. $88,560 \mathrm{~m}$; un autre affluent de l'Argent-Double, $2 \mathrm{~km}$ au $\mathrm{N}$. de Lespinassière, D. $88,800 \mathrm{~m}$.

Hérault : Prades-le-Lez, vers $150 \mathrm{~m}$; Saint-Gély-du-Fesc, vers $100 \mathrm{~m}$; l'Hérault (riv.) à Saint-Guilhem-le-Désert, vers $90 \mathrm{~m}$; l'Hérault à Ganges, vers $150 \mathrm{~m}$.

Gard : la Vis (riv.) au premier barrage en amont de Ganges, vers $200 \mathrm{~m}$; le Vigan, vers $300 \mathrm{~m}$.

\section{LISTE DES CAPTURES}

Abréviations. - L : larve; $\mathbf{N}$ : « nymphe », larve au dernier stade; $\mathbf{E}$ : exuvie larvaire.

Taeniopteryx schoenemundi (Mertens, 1923).

Espèce considérée comme probable pour le Massif Central par Aubert $|1963 \mathrm{a}|$.

Tarn : Albi, 19-II-1961, 1 ․

Aude : Brousse, 29-X-1962, 1 L.

Brachyptera risi (Morton, 1896).

Haute-Vienne : Pierre-Buffière, 14-IV-1963, $12 \mathrm{~N} \delta, 5 \mathrm{~N}$ \%

Puy-de-Dôme : Ceyrat, 20-V-1932, 1 ㅇ, 1 우.

Cantal : Rhue de Cheylade, 23-V-1964, 1 o, 1 \&.

Tarn : Célret 1, 18-III-1959, 2 N ô, $3 \mathrm{~N}$ \&, $4 \mathrm{E}$ \&; Céret 2, 18-III-1959, $2 \mathrm{~N} \hat{o}, 5 \mathrm{~N}$ q, $15 \mathrm{E}$; Anglès-du-Tarn, IV-1931, 2 우 ; le Sor, 14-IV-1963, $2 \mathrm{~N}$ \&; Sorèze, 4-IV-1963, $2 \mathrm{~L}$; Dourgne, 4-IV-1963, 2 ô, 3 \&, $43 \mathrm{~N}$ of,

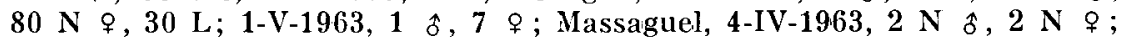

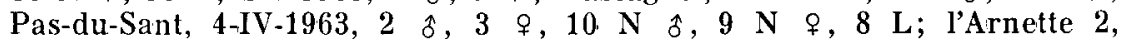
27-II-1963, $2 \mathrm{E}$ \&, $1 \mathrm{~L}$; ruisseau d'Albine 1, 29-IV-1963, $2 \mathrm{~N}$ ô, $3 \mathrm{~N}$ 우, $4 \mathrm{~L}$; ruisseau d'Albine 2, 29-IV $-1963,1$ ô ; ruisseau d'Albine 3, 29-IV1963,1 ․

Haute-Garonne : Saint-Ferréol, 9-IV-1931, 11 ô, 10 q, $2 \mathrm{~N} \hat{o}, 3 \mathrm{~N}$; 4-IV-1963, 1 of, $6 \mathrm{~N}$ \&, $6 \mathrm{~N}$ ㅇ, $29 \mathrm{~L}$.

Aude : Saint-Papoul, 16-IV-1963, $1 \mathrm{~N}$ 우 ; Ferrals, 16-IV-1963, 2 o, 10 ㅇ, $4 \mathrm{E}, 27 \mathrm{~N}$ ô, $52 \mathrm{~N}$ o, $82 \mathrm{~L}$; l'Alzeau, 24-IV-11963, 5 ô, 14 , $3 \mathrm{E}$; Brousse, 24-IV-1963, 1 q; le Clamoux 1, 13-II-1963, 12 L; Caunes, 13III-1963, $1 \mathrm{~N}$ ô; Citou, 13-H-1963, $1 \mathrm{~N}$ ô.

\section{Brachyptera seticornis (KLAPALEK, 1902).}

Cantal : Prat-de-Bouc, 22-V-1964, 1 ô.

Tarn : Forêt de Nore, 15-IV-1936, 19 $\hat{\delta}, 10 \%$; Sorèze, 4-IV-1963, $4 \hat{o}, 1$ ㅇ, $1 \mathrm{E}, 5 \mathrm{~N}$ oิ, $8 \mathrm{~N}$ ㅇ, $6 \mathrm{~L}$; Massaguel, 4-IV-1963, $5 \mathrm{~N}$ ô, $8 \mathrm{~N}$, 
$6 \mathrm{~L}$; Montimont 1, 1-V-1963, 2 우 ; l'Arnette 2, 27-IV-1963, 1 E ô, 1 L; ruisseau d'Albine 1, 29-IV-1963, $2 \mathrm{~N}$ ô, $3 \mathrm{~N}$ ㅇ, $1 \mathrm{~L}$; ruisseau d'Albine 2, 29-IV-1963, 1 ô; ruisseau d'Albine 4, 29-IV-1963, 2 우.

Haute-Garonne : Saint-Ferréol, 9-IV-1931, 3 ô, 2 우, $1 \mathrm{~N}$ ô, $1 \mathrm{~N}$ 우 4-IV-1963, $1 \mathrm{~N}$ ㅇ, $2 \mathrm{~L}$.

Aude : l'Orbiel, 1-V-1963, 2 E $\&$; le Clamoux 1, 13-II-1963, 3 L; Lespinassière, 29-IV-1963, $2 \mathrm{E}$ 우.

Brachyptera monilicornis (PICTET, 1842).

Espèce considérée comme probable pour le Massif Central par AUBERT [1963 a].

Tarn : Arfons, 13-II-1933, 1 \&.

Capnia nigra (Pictet, 1842).

Puy-de-Dôme : Besse, V-1958, 1 \& .

Cantal : Le Lioran, 2-V-1964, 1 o, 18 \&.

Capnia bifrons (NewMAN, 1838).

Puy-de-Dôme : Clermont-Ferrand, V-1931, 1 ô.

Aveyron : Rodez, 28-II-1949, 1 ô.

Tarn : Senouillac, 27-IV-1962, 1 \% ; le Clamoux 2, 13-H-1963, 1 N $\hat{o}$. Hérault : Prades-le-Lez, 18-IV-1930, 1 \%.

Capnioneura mitis Despax, 1932.

Tarn : Céret 2, 18-III-1959, 1 î, $1 \mathrm{~N}$ \% ; Anglès-du-Tarn, IV-1929, 1 ㅇ ; IV-1931, 1 ồ; Sorèze, 13-II-1933, 1 ô.

Haute-Garonne : Saint-Ferréol, 4-IV-1963, 2 ㅊ.

Aude : Ferrals, 16-IV-1963, 1 ๆ; Cabrespine, 3-XII-1962, 2 L; le Clamoux 1, 13-II-1963, 2 ô, 2 o, $3 \mathrm{~N}$ ô, $7 \mathrm{~N}$ \&, $2 \mathrm{~L}$; Caunes, 13-HI1963, 3 oิ, 5 ㅇ, $5 \mathrm{~N}$ ô, $5 \mathrm{~N}$ \%; Citou, 13-II-1963, $4 \mathrm{~N}$ ․

Protonemura praecox (Morton, 1894).

Puy-de-Dôme : Besse, IV-1958, 3 , \&, 3 ๆ.

Cantal : Le Lioran, 2-V-1964, 4 \%.

Aude : affluent de l'Argent-Double, 3-X-1962, 4 L.

Protonemura fumosa fumosa Ris, 1902.

Puy-de-Dôme : Vic-le-Comte, VIL1932, 1 î.

Protonemura fumosa spinulosa (NAvas, 1921).

Tarn : Montimont 1, 24-X-1962, 1 \&, 1 L; Les Rousses, 12-IX-1962,

$1 \mathrm{~N}$ \% ; l'Arnette 3, 27-II-1963, 1 L.

Aude : l'Arnette 1, 24-IV-1963, $3 \mathrm{~N}$ ô, $1 \mathrm{~N}$ ㅇ, $11 \mathrm{~L}$. 


\section{Protonemura meyeri (PICTET, 1841).}

Tarn : Sorèze, 4-IV-1963, 1 q; l'Arnette 2, 27-IV-1963, 1 ㅇ, $6 \mathbf{N}$ s, $2 \mathrm{~N}$ \%, $12 \mathrm{~L}$; ruisseau d'Albine 1, 29-IV-1963, 3 \%, $2 \mathrm{~N}$ \%, 1 L, ruisseau d'Albine 2, 29-IV-1963, 1 क ; ruisseau d'Albine 3, 13-IX-1962, 13 L; 29-IV1963, 5 $\hat{\delta}, 5$ ㅇ ; ruisseau d'Albine 4, 29-XI-1962, 5 L; 29-IV-1963, 2 ㅇ․

Haute-Garonne : Saint-Ferréol, 9-IV-1931, 2 ․

Aude : la Rigole, 29-X-1962, 5 I.; Brousse, 29-X-1962, 5 L; 4-IV-1963, 1 ㅇ ; Mas-Cabardès, 28-I-1963, $3 \mathrm{~N}$ q; Cabrespine, 3-X-1962, $7 \mathrm{~L}$; le Clamoux 1, 13-II-1963, $1 \mathrm{~N}$ of ; Caunes, 13-II-1963, $1 \mathrm{~N}$ \%

\section{Protonemura intricata Ris, 1902.}

Puy-de-Dôme : Ceyrat, 20-V-1932, 2 \&, 1 우.

Tarn : Anglès-du-Tarn, 19-V-1929, 1 ô; le Sor, 14-IV-19.63, $1 \mathrm{~N} \%$, $22 \mathrm{~L}$; Sorèze, 4-IV-1963, $10 \mathrm{~L}$; Dourgne, 4-IV-1963, $7 \mathrm{~L}$; Massaguel, 4łIV-1963, 25 L; Pas-du-Sant, 4-IV-1963, 1 ô, $3 \mathrm{~N}$ ô; Roquerlan, 27-II1963, $1 \mathrm{~L}$; Maison du Triby, 27-II-1963, $3 \mathrm{~L}$; ruisseau d'Albine 1, 29-IV$1963,4 \mathrm{~L}$.

Haute-Garonne : Saint-Ferréol, 22-IV-1931, 15 ô ; 22-VII-1931, 10 \&.

Aude : Ferrals, 16-IV-1963, $10 \mathrm{~N}$ \&, $6 \mathrm{~N}$ \%, $31 \mathrm{~L}$; l'Alzeau, 24-IV-1963, $1 \mathrm{~N}$ of, $2 \mathrm{~N}$ \%, $10 \mathrm{~L}$; l'Arnette 1, 24-IV-1963, 4 L; Cabrespine, 29-IV-1963, 1 of ; Citou, 29-IV-1963, 1 L.

\section{Protonemura beatensis (DEspax, 1929).}

Tarn : Montimont 1, 24-X-1962, $1 \mathrm{~N}$ is, $1 \mathrm{~N}$ \% Les Rousses, 12-IX$1963,3 \mathrm{~N}$ ô ; ruisseau d'Albine 3, 13-IX-1963, $2 \mathrm{~N}$ ㅇ, $2 \mathrm{~L}$.

Aude : l'Orbiel, 12-IX-1962, $1 \mathrm{~N}$ ô, $1 \mathrm{~N}$ ㅇ, $4 \mathrm{~L}$.

Protonemura nitida (PICTET) Ris, 1902.

Aveyron : Millau, 6-VI-1920, $1 \delta$.

Protonemura vercingetorix Aubert, 1963.

= Protonemura nimborum Despax [1951] nec RIs, 1902.

Puy-de-Dôme : Royat, V-1932, 1 ô, 1 ․

Cantal : Plrat-de-Bouc, 22-V-1964, 1 of, 21 q; Brezons, 22-V-1964, $1 \hat{o}$, 4 ㅇ․

Tarn : Forêt de Nore, 15-IV-1936, 1 ô ; Roquerlan, 27-II-1963, 2 N , 3 L; Maison du Triby, 27-II-1963, $2 \mathrm{~N}$ \%, ruisseau d'Albine 3, 29-IV1963,1 ô, 1 ․

Aude : Lespinassière, 29-IV-1963, $1 \hat{\text { ô. }}$.

Amphinemura sulcicollis (Stephens, 1835).

Cantal : Rhue de Cheylade, 23-V-1964, 3 of ; Velzic, 23-V-1964, 1 ô, 19.

Lozère : Dérols, 21-V-1964, 4 o, 12 ㅇ.

Tarn : le Sor, 14-IV-1963, $1 \mathrm{~N}$ ô ; Sorèze, 4-IV-1963, $5 \mathrm{~L}$; Massaguel, 4-IV-1963, $10 \mathrm{~L}$; ruisseau d'Albine 1, 29-IV-1963, $6 \mathrm{~N}$ \& , $5 \mathrm{~N}$ \&, $34 \mathrm{~L}$.

Haute-Garonne : Saint-Ferréol, V-1934, 1 ô. 
Amphinemura triangularis RIS, 1902.

Puy-de-Dôme : Saint-Saturnin, 22-V-1932, 2 ô, 2 ㅇ.

Amphinemura standfussi Ris, 1902.

Espèce considérée comme probable pour le Massif Central par Aubert $[1963 \mathrm{a}]$.

Tarn-et-Garonne : Miramont-de-Quercy, 2-IV-1931, 3 ô, 2 ㅇ․

Tarn : Senouillac, 3-V-1964, 2 \% .

Haute-Garonne : Saint-Ferréol, V-1934, 2 ô, 1 ㅇ. 3 L.

Aude : Saint-Papoul, 16-IV-1963, $8 \mathrm{~L}$; l'Alzeau, 24-IV-1963, 1 N ô,

Nemoura cinerea (Retzius, 1783).

Puy-de-Dôme : Besse, VII-1931, 2 ț, 1 q.

Cantal : Aurillac, 23-V-1964, 1 \&.

Lozère : Dérols, 21-V-1964, 5 f, 1 ; Salhiens, 21-V-1964, 1 ; Saint-Andéol, 21-V-1964, 2 ô, 1 우.

Aveyron : Rodez, 6-VI-1938, 1 \&.

Tarn-et-Garonne : Miramont-de-Quercy, 2-IV-1931, 2 \&.

Tarn : Gaillac, V-1964, 1 ; Les Rousses, 1-V-1963, 8 \& , 1 \&.

Haute-Garonne : Saint-Ferréol, 22-VII-1931, 2 \&.

Aude : Saint-Papoul, 16-IV-1963, 13 N, $21 \mathrm{I}$.

Gard : Le Vigan, 23-IV-1930, 12 o, 5 q.

Nemoura avicularis Morton, 1894.

Lozère : Dérols, 21-V-1964, 1 \%.

Tarn : Céret 1, 18-III-1959, 1 q, $1 \mathrm{~N} \hat{o}$.

Nemoura erratica ClaAssen, 1936.

= Nemoura risi Despax, 1929 a, 1934,

$=$ Nemoura erratica Kimmins [1940, 1950], Hynes [1940, 1958], Despax [1951], Aubert [1959, 1963 a],

nec Nemoura erratica Brinck [1949 a, 1949 b, 1952, 1956], Illies [1955], Müller-Liebenau [1961], Winkler [1957] selon Raušer [1965], KIS [1963].

Les indications ci-dessus ont été établies par l'examen de l'holotype de Nemoura erratica et de celui d'exemplaires de GrandeBretagne et de Suède, ainsi que grâce à des renseignements in litt. des $D^{\text {rs }}$ J. Illoes, de Plön, et B. Kıs, de Cluj. Il est probable que les autres citations de Nemoura erratica en Europe centrale et orientale [Pomeise 1961, Wojtas 1962, 1964, Botosaneand et Tabacaru 1963, repris par Miron 1964, Zhiltzova 1964] doivent aussi être rapportées à $N$. flexuosa. R. Sowa [1964] et J. RaušER [1964, 1965] n'ont trouvé que cette dernière espèce dans les Beskides et en Tchécoslovaquie. 
Comme nous l'avons déjà noté [Berthélemy 1960], l'armature de l'épiprocte, représentée par Aubert [1959], constitue le meilleur critère pour distinguer Nemoura erratica de $N$. flexuosa. Ce sont les figures des épiproctes données par BrincK $[1952,1956]$ qui nous ont suggéré que c'était $N$. flexuosa et non $N$. erratica qui avait été capturée en Suède.

Puy-de-Dôme : Besse, VII-1932, 1 ô; Royat, V-1932, 1 ô.

Cantal : Prat-de-Bouc, 22-V-1964, 3 ô, 6 \%; Brezons, 22-V-1964, 2 q.

Tarn : Forêt de Nore, 1-IV-1936, 14 ô, 5 \%; ruisseau d'Albine 4, 29-IV-1963, 1 o.

Haute-Garonne : Saint-Ferréol, 9-IV-1931, 1 ô, $10 q$.

Aude : le Lampy, 16-III-1932, 9 ô, 10 q.

Nemoura fulviceps KLAPALEK, 1902.

Tarn : Dourgne, 4-IV-1963, 1 ㅇ.

Nemoura marginata (PICTET) Ris, 1902.

Despax [1934, 1951] a discuté de la variabilité de cette espèce mais il est possible que certains des exemplaires qu'il avait eu en communication aient appartenu à Nemoura flexuosa. Je n'ai pas retrouvé dans sa collection ces spécimens, sans doute renvoyés à leurs propriétaires. Les mâles cités ci-dessous ont le bord interne des paraproctes légèrement échancré et plus sinueux que sur les figures de Ris .[1902], Despax [1951] et Aubert [1959]. Le même fait a été signalé par Moulıns [1962] pour des animaux capturés en Bourgogne. Les cerques et l'épiprocte sont, par contre, tout à fait conformes aux figures d'Aubert [1959] qui a d'ailleurs également récolté Nemoura marginata dans le Massif Central [AubErT 1963 a]. Il serait toutefois souhaitable d'étudier à nouveau la variabilité de $N$. marginata dans l'ensemble de son aire de répartition pour juger de l'opportunité de distinguer des sous-espèces.

Remarquons en outre qu'Aubert [1963 b] cite cette espèce des Pyrénées. Sans doute est-ce là une allusion à la mention « HauteGaronne à Gragnague »[DESPAx 1934, 1951]. Gragnague est en fait dans la région toulousaine, à $15 \mathrm{~km}$ environ au nord-est de Toulouse. A notre connaissance, Nemoura marginata n'a jamais été capturée dans les Pyrénées proprement dites et il s'agit d'une localisation erronée comparable à celle d'Isoperla ambigua (voir ci-dessous).

Tarn : Dourgne, 4-LV-1963, 1 of ; Les-Rousses, 1-V-1963, 1 \&.

Aude : Lespinassière, 29-IV-1963, 2 t, 6 ․

Nemoura flexuosa Aubert, 1949.

Lozère : Dérols, 21-V-1964, 3 ô, 7 \&; Saint-Andéol, 21-V-1964, 1 \&. 
Nemoura lacustris E. Picter, 1865.

= Nemoura monspessulana Despax, 1930.

Tarn : Dourgne, 4-IV-1963, 1 \&, 1 ㅇ.

Aude : Saint-Papoul, 16-IV-1963, 4 N, 3 L.

Hérault : Prades-le-Lez, 18-IV-1930, 8 \& , 9 \%, $2 \mathrm{~N}$; Saint-Gely-du-Fesc, 17-IV-1930, 33 \&, 32 \&, $8 \mathrm{~N}$ (localité type de Nemoura monspessulana).

Nemurella picteti KLAPALEK, 1909.

Cantal : Rhue de Cheylade, 23-V-1964, 1 우.

Aude : l'Arnette 1, 24-IV-1963, 1 L; Lespinassière, 29-IV-1963, 1 q.

Leuctra geniculata STEPhens, 1835.

Tarn : Castres, 24-IX-1947, 1 oิ, 4 오.

Aude : la Dure, 12-IX-1962, $1 \mathrm{~N}$; le Clamoux 2, 13-IX-1962, $3 \mathrm{~N}, 4 \mathrm{I}$.

Leuctra nigra OLIVIER, 1811.

Cantal : Prat-de-Bouc, 22-V-1964, 1 \& .

Leuctra fusca (LINNÉ, 1758).

Aveyron : Millau, 2 ô.

Tarn : ruisseau d'Albine 3, 13-IX-1962, $2 \mathrm{~N}$ \%

Aude : l'Alzeau, 29-X-1962, 1 \%, $1 \mathrm{~N}$; Brousse, 29-X-1962, 1 of, $10 \mathrm{~N}$; la Dure, 12-IX-1962, $9 \mathrm{~N}$; l'Orbiel, 12-IX-1962, $11 \mathrm{~N}$.

Leuctra major BRINcK, 1949.

Espèce considérée comme probable pour le Massif Central par A ubert [1963 a].

Aude : l'Argent-Double, 13-IX-1962, 1 L.

Leuctra carinthiaca sensu Mosely [1931, 1932] et Despax [1951].

Il semble bien que la question de la répartition et de la synonymie des Leuctra cingulata et L. carinthiaca de KEMPNy [1899] ne soit pas encore résolue de façon satisfaisante. A l'origine, KEMPNy a, en effet, établi ces deux espèces sur des couples mal appariés : le mâle décrit sous le nom de $L$. carinthiaca est une L. moselyi (voir Mosely 1931, 1932) tandis que la femelle décrite sous le nom de $L$. cingulata est une Leuctra dolasilla Consiguio, 1955 (voir Aubert 1959).

Or, à notre connaissance, des individus correspondant à la description du mâle de $L$. carinthiaca, rédigée par Mosely $[1931$, 1932] d'après des exemplaires des Vosges, n'ont jamais été capturés dans les Alpes. La véritable femelle de $L$. cingulata [AUbErt 1959] qui était restée inconnue de Mosely, est tout à fait conforme à la description de $L$. carinthiaca femelle. DEspax [1951] avait déjà 
signalé la présence dans la Grande Chartreuse de mâles de $L$. cingulata associés à des femelles qui « se rapprocheraient à tel point de carinthiaca que la distinction en serait presque impossible ».

A notre avis, le lectotype femelle de $L$. carinthiaca KEMPNy 1899, d'Autriche, est une femelle de $L$. cingulata. Quant à la « $L$. carinlhiaca » de Mosely, originaire des Vosges, c'est une espèce très voisine dont le mâle diffère de $L$. cingulata par le plus grand écartement des appendices du tergite VI et par les bords pigmentés cu tergite VIII qui ne sont pas dilatés vers l'intérieur en deux petites expensions dirigées vers l'arrière. La femelle de cette espèce est suffisamment proche de celle de $L$. cingulata pour que Mosely les ait confondues.

Les exemplaires cités ci-dessous correspondent en tous points à la description du mâle de «L. carinthiaca»par Mosely. Si notre hypothèse est exacte et si la «L. carinthiaca»de Mosely n'existe pas dans les Alpes, cette espèce devra recevoir un autre nom. Elle est jusqu'ici connue avec certitude des Vosges et du Massif Central et vit sans doute aussi dans le Sauerland (Ditrmar 1955].

Tarn : Forêt de Montaud, 8-X-1928, 2 ô; Montimont, 1, 24-X-1962, 1 o.

Leuctra despaxi Mosely, 1930.

Puy-de-Dôme : le Mont-Dore, 24-VI-1934, 1 ô, 1 \&.

Leuctra aurita Navas, 1919.

Puy-de-Dôme : Besse, VIII-1931, 2 \&, 1 \&.

Aude : la Loubatière, 8-X-1928, 3 ㅇ․

Leuctra castillana AubERT, 1956.

$=$ Leuctra pseudocylindrica Despax $1929 \mathrm{~b}, 1951$, pro parte.

La localité de la Loubatière, dans l'Aude, qui figure dans la description originale de Leuctra pseudocylindrica est erronée; il s'agit en fait de $L$. castillana et il est possible que la citation de L. pseudocylindrica pour le Mont-Dore [Mosely 1933, Despax 1951] soit également basée sur des exemplaires de cette espèce plutôt que sur des L. leptogaster comme le suggère Aubert [1963 a].

Aude : la I.oubatière, 8-X-1928, 1 ô, 5 우.

Leuctra hippopus KEMPNy, 1899. 1 ㅇ.

Cantal : la Rhue-de-Cheylade, 23-V-1964, 2 \&; Le Lioran, 2-V-1964,

Tarn : Anglès-du-Tarn, IV-1931, 2 \&, 4 \&; Forêt de Nore, 15-IV1936, $6 \hat{\delta}, 4$ क ; l'Arnette 2, 27-II-1963, $1 \mathrm{~N}$ ㅇ ; ruisseau d'Albine 1, 29IV-1963, 3 ô, 4 \%; ruisseau d'Albine 2, 29-IV-1963, 1 o, 4 \%; ruisseau 
d'Albine 3, 29-IV-1963, 21 $\delta, 17$; ruisseau d'Albine 4, 29-IV-1963, $2 \delta, 1$ \%

Haute-Garonne : Saint-Ferréol, 9-IV-1931, 6 o, 4 \&.

Aude : le Lampy, 16-III-1932, $6 \hat{\delta}, 3 \%$; l'Orbiel, 1-V-1963, 1 .

Leuctra prima KEMPNY, 1899.

Tarn : Mazamet, 15-IV-1936, $1 \delta, 1$; Maison-du-Triby, 27-II-1963, $1 \mathrm{~N}$ of.

Aude : Le Lampy, II-1936, 1 s , 1 \%.

Leuctra pseudosignifera AUBERT, 1954.

Cantal : Le Lioran, 2-V-1964, 1 \%.

Aude : Lespinassière, 29-IV-1963, 1 \%

Leuctra inermis KeMPNY, 1899.

Puy-de-Dôme : Saint-Saturnin, 22-V-1932, 2 o, 2 \%.

Cantal : Prat-du-Bouc, 22-V-1964, 4 o, 5 우 ; La-Rhue-de-Cheylade, 23$\mathrm{V}-1964,1$ \&.

Tarn : ruisseau d'Albine 3, 29-IV-1963, 15 $\delta, 9$; ruisseau d'Albinc, 4, 29-IV-1963, 4 \& , 4 \&.

Perlodes microcephala (PICTET, 1842).

Cantal : Le Lioran, 2-V-1964, 1 o; Prat-du-Bouc, 22-V-1964, 4 \&; Velzic, 23-V-1964, 1 \&.

Tarn : Sorèze, 4-IV-1963, $4 \mathrm{~N}$ \&; Montimont 1, 24-X-1962, 3 L; LesRousses, 12-IX-1962, $3 \mathrm{~L}$; Roquerlan, 27-II-1963, 1 L; ruisseau d'Albine 1 , 29-IV-1963, $4 \mathrm{~N}$ \% , $1 \mathrm{~L}$.

Aude : Ramondens, 24-X-1962, $3 \mathrm{~L}$; la Rigole, 29-X-1962, $1 \mathrm{~L}$; Brousse, 29-X-1962, $1 \mathrm{~L}$; la Dure, 12-IX-1962, 4 L; Mas-Cabardès, 28-I-1963, 2 L; l'Orbiel, 12-IX-1962, $6 \mathrm{~L}$.

Perlodes jurassica Aubert, 1946.

Cantal : Brézons, 22-V-1964, 2 ô, 3 \%; Prat-du-Bouc, 22-V-1964, 1 ô.

Isoperla grammatica (PODA, 1761).

Haute-Vienne : Saint-Vitte-sur-Briance, 12-VI-1962, 1 古.

Cantal : Murat, 14-Vili-1924, 3 o, 2 \%; Velzic, 23-V-1964, 7 ๙ , 11 ; Saint-Julien-de-Jordanne, 23-V-1964, 2 o, 5 \&; Aurillac, 23-V-1964, 1 o; Saint-Simon, 23-V-1964, 3 ; Arpajon-sur-Cère, 24-V-1964, $1 \hat{\delta}, 4 q$; Carbonnat, 24-V-1964, 5 \& 7 \% .

Lozère : Mende, 28-VII-1924, 1 q Dérols, 21-V-1964, $4 \hat{\delta}, 4$ q.

Tarn : Senouillac, 5-IV-1961, 2 \&; 17-IV-1961, 1 \%; 3-V-1964, 1 ; Dourgna, 4-IV-1963, 2 L, $1 \mathrm{E}$.

Haute-Garonne : Saint-Ferréol, 2-IV-1932, 1 ô.

Aude : Saint-Papoul, 16-IV-1963, 1 L; Ferrals, 16-IV-1963, $2 \mathrm{~N}$ o, $4 \mathrm{~N}$ \&, $29 \mathrm{~L}$; l'Alzeau, 24-IV-1963, $1 \mathrm{~N}$; le Cros, 29-IV-1963, $1 \mathrm{~N}$ of, 4 L. Hérault : Ganges, 19-V-1930, 5 \&, 4 क (types d'Isoperla grammatica Iriangularis DESPAX, 1936). 
Isoperla oxylepis (DESPAX, 1936).

Haute-Vienne : Saint-Vitte-sur-Briance, 12-VI-1962, 1 ô.

Cantal : Saint-Julien-de-Jondanne, 23-V-1964, 1 ; ; Velzic, 23-V-1964, 3 s, 13 \%.

Isoperla acicularis (DESPAX, 1936).

Cantal : La-Rhue-de-Cheylade, 23-V-1964, 3 \&.

Isoperla ambigua (DESPAX, 1936).

Le sac pénial du mâle récolté au cirque du Brézons présente latéralement deux petits amas de spicules moins fortement chitinisés que ceux de l'armature principale mais qui tranchent assez nettement, néanmoins, sur l'ornementation générale de la paroi. ILLIEs [1952], qui mettait en doute la valeur spécifique de cette forme, note d'autre part que l'armature accessoire d'Isoperla rivulorum est parfois très réduite. Les citations de Festa [1942, 1949] pour I'Italie sont rapportées à Isoperla grammatica et I. rivulorum par Aubert [1958], mais Consiglio [1962] attribue à 1 . ambigua des Isoperla récoltées en Ligurie et a décrit, en 1961, une forme voisine originaire de Sicile.

Il existe donc çà et là des formes étroitement apparentées à Isoperla rivulorum, mais qui en diffèrent par l'absence d'armatures accessoires. Il n'est pas possible, à l'heure actuelle, de savoir si ces formes ont une origine monophylétique et, en attendant des comparaisons directes entre larves et adultes de différentes provenances, il paraît préférable de considérer Isoperla ambigua comme endémique du Massif Central.

Les données d'Aubert [1946], Illlies [1952] et Consiglio [1961] qui citent $I$. ambigua des Pyrénées sont inexactes. I. ambigua n'y a jamais été récoltée jusqu'ici, à notre connaissance. Bien que situé en Haute-Garonne, Saint-Ferréol est à l'extrémité sud-ouest de la Montagne Noire. La forme très allongée du département de la Haute-Garonne, qui prend en écharpe des régions géographiques aussi distinctes que la bordure du Massif Central, le bassin souspyrénéen, les Petites Pyrénées, la dépression prépyrénéenne et les Pyrénées centrales, est sans doute à l'origine de cette localisation erronée.

Puy-de-Dôme : Mont-Dore, 24-VI-1934, 3 ô, 2 ㅇ․

Cantal : Brézons, 22-V-1964, 1 \& ; La-Rhue-de-Cheylade, 23-V-1964, 1 s.

Haute-Garonne : Saint-Ferréol, 22-VI-1931, 1 ô, 1 ㅇ․

Perla marginata (Panzer, 1799).

Cantal : Velzic, 23-V-1964, 3 ô.

Tarn : Anglès-du-Tarn, 1 ô, 1 †; Sorèze, 4-IV-1963, $1 \mathrm{~N}$ \&, $1 \mathrm{~L}$; Mas- 
saguel, 4-IV-1963, 11 L; Montimont 2, 12-IX-1962, 13 L, 1 E; ruisseau d'Albine 2, 13-IX-1962, $5 \mathrm{~L}$.

Haute-Garonne : Saint-Ferréol, 9-IV-1931, 3 L.

Aude : la Rigole, 29-X-1962, 3 L; Cabrespine, 3-XII-1963, 1 L; le Cros, 29-IV-1963, $1 \mathrm{~L}$; affluent de l'Argent-Double, 3-X-1962, 2 L.

Hérault : Ganges, 19-V-1930, 1 L, 1 E.

Perla burmeisteriana ClaAssen, 1936.

Haute-Vienne : Sainte-Vitte-sur-Briance, 12-VI-1962, 1 \%.

Puy-de-Dôme : Vic-le-Comte, 17-V-1931, 1 ㅇ, 2 ㅇ.

Aveyron : Rodez, 5-VI-1938, 2 \&.

\section{Dinocras cephalotes (CurTIS, 1827).}

Tarn : Massaguel, 4-IV-1963, 15 L; Pas-du-Sant, 4-IV-1963, 1 L; Montimont 2, 12-IX-1962, $1 \mathrm{~L}, 3 \mathrm{E}$; l'Arnette 4, 12-IX-1962, $1 \mathrm{~L}$; Roquerlan, 27-II-1963, 7 L; ruisseau d'Albine 2, 13-IX-1962, $10 \mathrm{~L}$; ruisseau d'Albine 3, 13-IX-1962, $29 \mathrm{~L}$; ruisseau d'Albine 4, 26-XI-1962, $10 \mathrm{~L}$.

Haute-Garonne : Saint-Ferréol, 9-IV-1931, 1 L.

Aude : la Rigole, 29-X-1962, 3 L; l'Orbiel, 12-IX-1962, 1 L; l'ArgentDouble, 13-IX-1963, $1 \mathrm{~L}$.

\section{Chloroperla torrentium (PICTET, 1842).}

Haute-Vienne : Saint-Vitte-sur-Briance, 12-VI-1962, 15 \&ิ, 5 \&.

Puy-de-Dôme : Mont-Dore, 24-V-1934, 4 \% $10 \%$ (forme manevali Kimmins, 1935).

Cantal : Murat, 14-VIII-1924, 1 ô, 1 ; Le Lioran, 9-VII-1924, 13 of, 18 \&, Brezons, 22-V-1964, 4 \& (dont une forme manevali) La-Rhue-deCheylade, 23-V-1964, 1 ; Saint-Julien-de-Jordanne, 23-V-1964, 2 ; Velzic, 23-V-1964, 1 ㅇ.

Lozère : Dérols, 21-V-1964, 11 \& , 12 ․

Tarn : Massaguel, 4-IV-1963, $1 \mathrm{~L}$; Roquerlan, 27-II-1963, $1 \mathrm{~L}$.

Aude : la Rigole, V-1936, 2 ; ; Ramondens, 24-X-1962, 1 L.

Hérault : Saint-Guilhem-le-Désert, 10-VI-1930, 3 ㅇ, 1 오.

Gard : en amont de Ganges, 19-V-1930, 2 s, 3 ㅇ.

Chloroperla tripunctata (Scopoli, 1763).

Cantal : Murat, 14-VII-1924, 1 $\hat{\delta}, 1$ \&; Saint-Julien-de-Jordanne, 23-V1964,1 ; ; Velzic, 23-V-1964, 1 o, 7 \%.

\section{CONCLUSION}

Nous avons donné ci-dessus la répartition détaillée de plus de 2000 Plécoptères, comportant 48 espèces. Seules 4 d'entre elles, Taeniopteryx schoenemundi, Brachyptera monilicornis, Amphinemura standfussi et Leuctra major n'avaient pas été rencontrées par Auber T [1963 a] qui les considérait simplement comme probables pour le Massif Central. Ce fait confirme la grande homogénéité du 
peuplement, puisque les récoltes étudiées ici ont été faites le plus souvent dans des régions différentes. D'autre part, on peut admettre que l'on connaît maintenant une importante proportion de la faune totale. Comparé à celui des régions les mieux connues au point de vue faunistique, le nombre d'une soixantaine d'espèces de Plécoptères pour le Massif Central est en accord avec la variété des biotopes offerts et il ne s'accroîtra sans doute à l'avenir que d'assez peu d'unités.

Aussi pouvons-nous envisager dès maintenant les relations du Massif Central avec l'Europe moyenne, d'une part, et avec les Pyrénées, d'autre part. Les deux tiers des espèces du Massif Central vivent aussi dans ces deux autres régions. Ce sont : Taeniopteryx schoenemundi, T. hubaulti, Brachyptera risi, B. seticornis, B. monilicornis, Capnia nigra, C. bifrons, C. vidua, Capnioneura mitis, Protonemura praecox, P. meyeri, $P$. intricata, Amphinemura sulcicollis, A. triangularis, A. standfussi, Nemoura cinerea, $N$. avicularis, $N$. erratica, $N$. fulviceps, $N$. flexuosa, Nemurella picteti, Leuctra geniculata, L. digitata, L. fusca, L. aurita, L. major, L. leptogaster, L. albida, L. hippopus, L. prima, L. inermis, Perlodes microcephala, Isogenus nubecula, Isoperla grammatica, Perla marginata, $P$. burmeisteriana, Dinocras cephalotes, Chloroperla torrentium, Ch. tripunctata. Comme le suggère Aurert [1963 a], il faudra sans doute leur ajouter Leuctra mortoni et Dictyogenus imhoffi qui vivent en Europe moyenne et dans les Pyrénées dans les biotopes dont les équivalents existent aussi dans le Massif Central.

Dix autres sont communes à l'Europe moyenne et au Massif Central mais n'ont encore jamais été rencontrées dans les Pyrénées. Ce sont : Rhabdiopteryx neglecta, Protonemura fumosa fumosa, $P$. nitida, P. montana, Nemoura marginata, Leuctra nigra, L. carinthiaca, L. pseudosignifera, Perlodes jurassica, Isoperla oxylepis.

Cinq autres vivent dans les Pyrénées et le Massif Central mais sont inconnues d'Europe moyenne. Ce sont : Protonemoura fumosa spinulosa, P. beatensis, Leuctra despaxi, L. castillana et Isoperla acicularis.

Enfin, sur les quatre formes endémiques du Massif Central, deux sont voisines d'espèces d'Europe moyenne : Protonemura vercingetorix est proche de $P$. nimborum, avec laquelle elle avait été confondue par Despax, et Isoperla ambigua d'Isoperla rivulorum. Leuctra flavomaculata, par contre, présente de grandes affinités avec une Leuctra pyrénéenne, $L$. alosi, et on pourrait admettre qu'il s'agit d'une sous-espèce [Aubert 1957]. La quatrième, enfin, Leuctra gallica, est voisine de L. hippopus qui se rencontre à la fois en Europe moyenne et dans les Pyrénées. 
Il y a donc deux fois plus d'espèces communes à l'Europe moyenne et au Massif Central qu'à ce dernier et aux Pyrénées. Nous ne pensons pas que la faune des Plécoptères pyrénéens soit insuffisamment connue au point que cette différence puisse s'expliquer simplement par un degré d'investigation moins élevé. Il nous semble plutôt que la dépression de Carcassonne a opposé à la dissémination des Plécoptères une barrière deux fois plus efficace que l'extrémité Sud-Est du Bassin de Paris. Cette conclusion est en parfait accord avec les données altitudinales et surtout climatiques. Outre la recherche d'éventuelles formes de passage entre Protonemura fumosa fumosa et $P$. $f$. spinulosa le long de leur ligne de contact, le principal point restant à préciser est donc l'extension vers le Nord-Est, dans le Morvan et la Bourgogne, des formes pyrénéennes inconnues en Europe moyenne.

Faunistic notes on the Stoneflies of the French Massif Central (Plecoptera).

The detailed distribution of more than two thousand Stoneflies, belonging to forty eight species and coming mainly from the South Western region of the Massif Central, is given in the French text. Only four of them, Taeniopteryx schoenemundi, Brachyptera monilicornis, Amphinemura standfussi and Leuctra major, had not previously been found by Aubert [1963 a].

Forty of the fifty nine Stoneflies known from the Massif Central live also in both Central Europe and the Pyrenees. Ten of them live in Central Europe but are not yet known in the Pyrenees (Rhabdiopteryx neglecta, Protonemura fumosa fumosa, $P$. nitida, $P$. montana, Nemoura marginala, Leuctra nigra, L. carinthiaca, L. pseudosignifera, Perlodes jurassica, Isoperla oxylepis). In contrast, five are Pyrenean species (Protonemura fumosa spinulosa, P. beatensis, Leuctra despaxi, L. castillana, Isoperla acicularis), absent in Central Europe. Two of the endemic Stoneflies, Protonemura vercingetorix and Isoperla ambigua, are related to Central European forms (Protonemura nimborum and Isoperla rivulorum), and one, Leuctra flavomaculata, to the Pyrenean $L$. alosi.

It seems therefore that the ecological barrier of the Depression of Carcassonne, in the South of the Massif Central, has acted twice as effectively as the Plateau de Langres to prevent the dissemination of Stoneflies.

\section{Faunistische Untersuchungen über den Plecopteren des fRAnzösischen Massif Central.}

Ausführliche Angaben über die Verbreitung von mehr als 2000 . Plecopteren, die zu 48 verschiedenen Arten gehören, und die vor allem im südwestlichen Teil des Massif Central gesammelt worden waren, werden im französischen Text gegeben. 4 von ihnen, Taeniopteryx 
schoenemundi, Brachyplera monilicornis, Amphinemura standfussi und Leuctra major, waren vorher von AuBERT [1963 a] noch nicht gefunden worden. 40 von 59 Arten, die im. Massif Central bekannt sind, leben sowohl in Mitteleuropa als auch in den Pyrenäen. 10 von ihnen leben in Mitteleuropa, sind aber noch unbekannt in der Pyrenäen (Rhabdiopte$r y x$ neglecta, Protonemura fumosa fumosa, $P$. nitida, $P$. montana, Nemoura marginala, Leuctra nigra, L. carinthiaca, L. pseudosignifera, Perlodes jurassica, Isoperla oxylepis). Dagegen fehlen 5 pyrenäische Arten in Mitteleuropa (Protonemura fumosa spinulosa, P. beatensis, L. despaxi, L. castillana, Isoperla acicularis). 2 der endemischen Formen sind verwandt mit anderen in Mitteleuropa (Protonemura vercingetorix mit P. ni.mborum und Isoperla ambigua mit $I$. rivulorum), eine mit der pyrenäischen Leuctra alosi (L. flavomaculata).

Es hat also den Anschein, dass die Bodensenke von Carcassonne südlich des Massif Central ein ökologisches Hindernis gebildet hat, die doppelt so wirkungsvoll ist wie die Hochebene von Langres für die Verbreitung der Plecopteren.

\section{TRAVAUX CITÉS}

Aubert (J.). 1946. - Les Plécoptères de la Suisse romande. Mitt. Schweiz. Ent. Ges., 20 : 7-128.

Aubert (J.). 1957. - Les Leuctra du groupe inermis KEMPNy et quelques espèces inermes isolées (Plécoptères Leuctridae). Mitt. Schweiz. Ent. Ges., 30 : 285-312.

Aubert (J.). 1958. - Les Plécoptères de Calabre (Italie méridionale). Ann. Ist. Mus. Zool., Univ. Napoli, 10, n: 4 : 1-52.

Aubert (J.). 1959. - Plecoptera. Insecta helvetica Fauna, 1 : 140 p.

Aubert (J.). 1963 a. - Contribution à l'étude des Plécoptères du Massif Central. Bull. Soc. Ent. France, 68 : 163-185.

Aubert (J.). 1963 b. - Les Plécoptères de la péninsule ibérique. Eos, 39 : 23-107.

Berthélemy (C.). 1960. - Note sur quelques Nemouridac (Plécoptères) du Sud-Ouest de la France. Bull. Soc. Zool. France, 85, ${ }^{\circ} 1$ : $52-58$.

Botosaneanu (L.) et Tabacaru (I.). 1963. - Ephéméroptères, Plécoptères et Trichoptères des Monts de Fagarasch (Alpes de Transylvanic). Inst. r. Sc. nat. Belgique, Bull., 39, $\mathrm{n}^{\circ} 38: 58 \mathrm{p}$.

Bhinck (P.). 1949 a. - Studies on Swedish Stoneflies (Plecoptera). Opusc. Ent., Suppl. XI : 250 p.

Brinck (P.). 1949 b. - Catalogus Insectorum Sueciae X. Plecoptera. Opusc. Ent., $1949: 177-183$.

Brinck (P.). 1952. - Bäcksländor, Plecoptera. Swensk Insektfauna, 15 : $128 \mathrm{p}$.

Brinck (P.). 1956. - Reproductive system and mating in Plecoptera. Opusc. Ent., 21 : 57-128.

Consigrio (C.). 1961. - Plecotteri di Sicilia e d'Aspromonte e classificazione delle Isoperla Europee. Mem. Mus. Civ. St. Nat. Verona, 9 : 173-196. 
Consiglio (C.). 1962. - Contributo alla conoscenza dei Plecotteri del Piemonte, Valle d'Aosta e Liguria. Mem. Soc. Entam. Ital., 41 : 25-44.

Despax (R.). 1929 a. - Plécoptères pyrénéens I. Étude et description de quelques formes du genre Nemura Latr. Bull. Soc. Hist. nat. Toulouse, 58 : 77-104.

Despax (R.). 1929 b. - Plécoptères pyrénéens II. Étude et description de quelques formes du genre Leuctra STEPH. Bull. Soc. Hist. Nat. Toulouse, 58 : 165-178.

Despax (R.). 1930. - Nemura monspessulana nov. sp., Plécoptères nouveau du Sud de la France. Bull. Soc. Hist. Nat. Toulouse, 60 : 233-237.

Despax (R.). 1934. - Plécoptères pyrénéens VIII. Etude et description de quelques formes de Niémoures apparentés à Nemoura marginata Pict. Bull. Soc. Hist. Nat. Toulouse, 66 : 255-270.

Despax (R.). 1936. - Contribution à l'étude du genre Chloroperla PicTET (Isoperla Banks). Bull. Soc. Hist. Nat. Toulouse, 69 : 337-398.

Despax (R.). 1951. - Plécoptères. Faune de France, 55 : 280 p.

Dittmar (H.). 1955. - Ein Sauerlandbach. Arch. f. Hydrobiol., 50, $3 / 4: 305-552$.

Festa (A.). 1942. — Studi sui Plecotteri italiani. VI. Plecotteri raccolti dal Dott. Felice Capra in val Chiobbia. Bol. Soc. Ent. It., 74 : 61-63.

Festa (A.). 1949. - Studi sui Plecotteri italiani. IX. Nuovi reperti. Bol. Soc. Ent. It., 79 : 32-34.

Hynes (H. B. N.). 1940. - A key to the British species of Plecoptera (Stoneflies) with Notes on their Ecology. Freshw. biol. Ass. Brit. Emp. Sci. Publ., 2 : 39 p.

HyNes (H. B. N.). 1958. - A key to the adults and nymphs of British Stoneflies (Plecoptera). Freshw. biol. Ass. Brit. Emp. Sci. Publ. $17: 88 \mathrm{p}$.

ILLIES (J.). 1952. - Die europäischen Arten der Plecopterengattung Isoperla Banks (= Chloroperla PICTET). Beitr. z. Ent., 2 : 369-424.

ILLIES (J.). 1955. - Steinfliegen oder Plecoptera. Die Tierwelt Deutschlands, $43: 150 \mathrm{p}$.

Kempny (P.). 1899. - Zur Kenntnis der Plecopteren, III. Neue und ungenügend bekannte Leuctra-Arten, II. u. III Teil. Verh. $k$. $k$. zool. bot. Ges. Wien, $49: 9-15,269-278$.

KIMmins (D. E.). 1935. - A new Brachypterous Isopteryx from France (Plecoptera). Ann. Magaz. Nat. Hist., Ser. 10, 15 : 645-650.

Kimmins (D. E.). 1940. - A synopsis of the British Nemouridae. Trans. Soc. Brit. Ent., 7 : 65-83.

Kimmins (D. E.). 1950. - Plecoptera. Handbooks for the Identification of British Insects, 1 : $\mathrm{n}^{0} 6,18 \mathrm{p}$.

KIs (B.). 1963. - Zur Kenntnis der Plecopteren-Fauna Rumäniens. Fol. Entom. Hungar., 16, $\mathrm{n}^{\circ} 3: 67-82$.

Mrron (I.). 1964. - Beiträge zum Studium der Steinfliegen (Plecoptera) der Ostkarpaten. Gewässer und Abwässer, 34/35 (Verh. 3. Int. Symp. Plecopteren) : 81-92. 
Mosely (M. E.). 1931. - The identity of Leuctra carinthiaca KemPNy (Plecoptera). Ann. Mag. Natur. Hist., 10, $7: 341-343+1 \mathrm{pl}$.

Mosery (M. E.). 1932. - A revision on the European Species of the Genus Leuctra (Plecoptera) Ann. Mag. Natur. Hist., 10, 10 : $1-41+5$ p'l.

Mosely (M. E.). 1933. - The Trichoptera and Plecoptera of the Auvergne region of France. Entomologist, 66 : 112-117.

Mourins (M.). 1962. - Contribution à l'étude de la faune de France des Plécoptères (Bourgogne, Basses-Alpes). Trav. Lab. Zool. Stat. Aquic. Grimaldi Fac. Sc. Dijon, $44: 28$ p.

Müller-Liebenau (I.) . 1961. - Steinfliegen aus der Eifel (Insecta, Plecoptera). Gewässer und Abwässer, 29 : 41-55.

Pomeisl (E.). 1961. - Ordnung : Plecoptera, in Franz (H.), die Nordost-Alpen im Spiegel ihrer Landtierwelt, eine Gebietsmonographie. Bd 2 : 56-73.

RaušER (J.). 1964. — Verbreitungsgeschichte der tschechoslowakischen Plecopterenassoziationen. Gewässer und Abwässer, 34/35 (Verh. 3. Int. Symp. Plecopteren) : 115-129.

Raušer (J.). 1965. — Plécoptères nouveaux pour la Tchécoslovaquie. Mitt. Schweiz. Ent. Ges., 37 (1964), n³ : 157-163.

Ris (F.). 1902. -- Die schweizerischen Arten der Perlidengattung Nemura. Mitt. Schweiz. Ent. Ges., 10 : 378-406, 432.

Sowa (R.). 1964. - Drei interessante Arten der Nemoura-Gattung (Plecoptera in Polen. Bull. Acad. Polon. Sc. Cl. II, 12, n ${ }^{\circ} 8: 347-349$.

Winklek (O.). 1957. - Plecoptera slovenska (Faunisticko-systematicka studia). Biologické prace, $3, \mathrm{n}^{\circ} 7: 1-96$.

IVo.JTas (F.). 1962. - Widelnice (Plecoptera) rzeki Grabi. Lodzkie Tow. Nauk. Wydz. III, 7y : 28 p.

WoJtas (F.). 1964. - Die Plecopterenfauna Polens mit berücksichtigung des Tatragebiets. Gewässer und Abwässer, $34 / 35$ (Verh. 3. Int. Symp. Plecopteren). : 93-100.

Zhiltzova (L. A.). 1964. - Die Plecopteren des europäischen teils der Sowjetunion und des Kaukasus. Gewässer und Abwässer, 34/35 (Verh. 3. Int. Symp. Plecopteren) : 101-114.

(Laboratoire de Zoologie, Faculté des Sciences, 118, route de Narbonne, Toulonse). 